\title{
IMPACT OF IRRIGATION FREQUENCIES, POTASSIUM FERTILIZER AND VITAMIN TREATMENTS ON YIELD, NUTRIENTS AND AMINO ACID CONTENTS OF BREAD WHEAT GRAINS
}

\author{
EL-AREF, $\mathrm{KH}^{1}$.A, M. A. FARAGALLAH ${ }^{2}$ AND S. A. DESOUKY ${ }^{3}$ \\ 1-Agronomy Dep., Fac. Agric., Al-Azhar Univ. Assiut, Egypt. \\ 2-Soil and water Dep., Fac. Agric., Al-Azhar Univ. Assiut, Egypt. \\ 3-Botany and Micro. Dep., Fac. Sci, Al-Azhar Univ. Assiut, Egypt.
}

\begin{abstract}
This study is conducted to investigate the effect of irrigation intervals in combination with soil application and / or foliar spray of potassium fertilizer and soaking in some vitamins (ascorbic acid and pyridoxine) on wheat yield, certain nutrients and amino acid contents in grains, as well as field water use efficiency. The main aim of the study is to save of the irrigation water without reduction of wheat yield with facility of practical application. Two field experiments were carried out during the two successive winter seasons of 2005/2006 and 2006/2007 at the experimental farm, Faculty of Agriculture, Al-Azhar University at Assiut Governorate. The obtained results are as the follows:
\end{abstract}

The grain yield and its components were significantly affected by the main treatments i. e. irrigation frequencies, vitamins and potassium fertilizer. The irrigation every 21-day interval gave higher values of grain yield and all studied characters as compared with the other irrigation frequencies (28-day and 35- day intervals) in two seasons. The soaking in pyridoxine gave higher values of grain yield and all other characters as compared with control and ascorbic acid in both seasons. The superior grain yield of wheat per Feddan which resulted from treatment with potassium as foliar spray during growing seasons was applied.

The interaction effects between irrigation frequencies, every 21-day and soaking in pyridoxine vitamin gave higher values of grain yield of wheat and all other characters only except harvest index as compared with the other treatments in both seasons. The highest values of grain yield of wheat and all other characters were obtained when irrigation every 21 day and application of all potassium as foliar spray in both seasons.

The highest values of the grain yield and its components were obtained when irrigation was applied every 21-day, with soaking in pyridoxine vitamin and application of potassium fertilizer as foliar spray in both seasons. However, the lowest values of the grain yield and its components were recorded by applied irrigating the plants every 35-day, soaking in water and without potassium fertilizer in 2005/2006 and 2006/2007 seasons.

The interactions of irrigation interval $\mathrm{x}$ vitamins $\mathrm{x}$ potassium application significantly affected the macronutrients and total amino acid contents in wheat grains. Irrigation every 28day interval with soaking in ascorbic acid and potassium application as foliar spray realized the highest values of nitrogen and phosphorus and total amino acid contents in wheat grains. Also, the highest amounts of potassium content in wheat grain were found under the same treatment but without soaking in any vitamins (soaking in water).The field water use 
efficiency increased when moisture stress increased. The highest value of field water use efficiency was calculated when plant irrigated at 35-day interval with soaking in pyridoxine and foliar sprayed with potassium.

Keyword: irrigation frequencies, vitamins, potassium fertilizer, grain yield, macronutrients and total amino acids.

\section{Introduction}

In all countries of the world, water is considered a main limiting factor in agricultural expansion. In Egypt, Wheat (Triticum aestivum L.) is considered a major feed crop. With over growing population, increasing wheat productivity with decreasing water consumption is a national target. In this context, many studies were performed to increase of wheat yield and improve its quality, as well as increase water consumptive use efficiency through popper fertilization and good management. Similarly, soil moisture is one of the factors, which influence the yield and quality of crops. Thus, scheduling irrigation for wheat plant may improve its production per unit area and / or consumed water unit.

Potassium is considered as an essential element in nearly all processes needed to sustain growth, reproduction and metabolic activities of plants, such as photosynthesis, protein synthesis, and ion balance control, regulation opening and closing of plant stomata and water uses (Marschner, 1995). It has been mentioned that, potassium application play an important role in plants grown under water deficit. Since, potassium fertilization slightly decreased daily and monthly actual water consumptive use. Moreover, foliar spray of potassium ensures the quick and adequate K- supply for plants at the time of yield formation to improve its productivity (Talha et al., 1987; El Yamany, 1994; Abdel - Hafez et al., 1999; Hussien et al., 2000; Abdel-Aziz et al., 2004; El-Saei et al., 2006).

El-Saei et al., (2006) found that, the values of wheat yield and field water use efficiency increased with increasing the rate of potassium fertilizer. Also, AbdelAziz et al., (2004) found that, gradual and significant increases of maize productivity as a result of the reduction in soil moisture depletion. The same response was observed for raising $\mathrm{K}$ - level up to $3 \%$ as foliar spray. On the other hand, Abd El-Gawad et al. (1993) reported that when wheat plants were subjected to water stress at any physiological stage depressed yield and yield attributes.

Irrigation every 10-days or 20-days interval increased significantly the yield of components and growth characters of sorghum cultivar. Moreover, the highest 
values of grains and $\mathrm{N}$ - recovery in grains could obtained under any applied of $\mathrm{N}$ fertility level at irrigation every 10-days or 20-days interval, while the lowest values were recorded at irrigation every 30- days interval (Ragheb and Elnagar, 1997). Also, Ibrahim et al. (1992) reported that leaf area of plant and grain yield (ard /Fed) of maize plant were significantly increase with the decreased in irrigation period.

Vitamins are considered as triggers and cofactors in metabolism of plant cells. In this context, many attempts have been performed to counteract the noxious effect of drought stress on growth and physiological activities of plants. By using various vitamins such as ascorbic acid, and pyridoxine on Lupinus termis, Vigina sinensis and Vicia faba plants grown under normal and stress conditions, Azooz (1990) and El-Tayeb (1991) stated that these vitamins were important for growth of plants under stress conditions. Presowing soaking of seeds in either ascorbic acid or pyridoxine significantly stimulated the root and shoot length (Shaddad et al. 1990).

To attempt spare irrigation of water without reduction of wheat yield and in the same time facility of practical application. Thus, the present study is conducted to study the effect of irrigation intervals in combination with soil application and / or foliar spray of potassium fertilizer and soaking in some vitamins (ascorbic acid and pyridoxine) on wheat yield, certain nutrients and amino acid contents in grains, as well as field water use efficiency.

\section{Materials And Methods}

Two field experiments were carried out during the two successive winter seasons of 2005/2006 and 2006/2007 at the experimental farm, Faculty of Agriculture, AlAzhar University at Assiut Governorate. Some soil physical and chemical characteristics of soil of the experimental site were determined according to methods of Page (1982) and Klute (1986) as shown in table (1). Giza - 168 cultivar of wheat ( Triticum aestivum L.) was chosen for this study, where it is the most commonly used cultivar by farmers in Upper Egypt .The experimental design was split- split plot design with three replicates. The replicate includes 36 plots combination between used treatments. The area of each plot was $10.5 \mathrm{~m}^{2}\left(3.5 \mathrm{X} 3 \mathrm{~m}^{2}\right)$ with area of $1 / 400 \mathrm{Fed}$. Irrigation frequency was fixed in the main plots, the vitamins soaking (ascorbic acid and pyridoxine $200 \mathrm{ppm}$ ) in the sub -plots and the potassium application in the sub-sub-plots. To avoid the interference between irrigation treatments, $3 \mathrm{~m}$ beds were left between the main plots. 
Table (1): Some physical and chemical properties of the studied soil.

\begin{tabular}{|c|c|c|c|}
\hline \multirow{2}{*}{ Characteristics } & \multicolumn{3}{|c|}{ Depth ( cm) } \\
\hline & $0-30$ & $30-60$ & $60-90$ \\
\hline Sand & 26.30 & 25.80 & 25.30 \\
\hline Silt & 38.00 & 38.50 & 39.50 \\
\hline Clay & 35.70 & 35.70 & 35.20 \\
\hline Texture grade & Clay loam & Clay loam & Clay loam \\
\hline Organic matter $\%$ & 1.25 & 0.95 & 0.73 \\
\hline $\mathrm{CaCO}_{3} \%$ & 3.45 & 2.60 & 2.25 \\
\hline $\mathrm{pH}(1: 2.5)$ & 8.25 & 8.50 & 8.55 \\
\hline $\mathrm{EC}(\mathrm{ds} / \mathrm{m} 1: 5)$ & 0.25 & 0.28 & 0.30 \\
\hline \multicolumn{4}{|c|}{ Soluble Cations ( $\mathrm{mg} / \mathrm{l})$} \\
\hline $\mathrm{Ca}^{+2}$ & 0.55 & 0.40 & 0.30 \\
\hline $\mathrm{Mg}^{+2}$ & 0.50 & 0.60 & 0.40 \\
\hline $\mathrm{Na}^{+}$ & 1.50 & 1.55 & 1.65 \\
\hline $\mathrm{K}^{+}$ & 0.04 & 0.03 & 0.02 \\
\hline \multicolumn{4}{|l|}{ Soluble Anion( $\mathrm{mg} / \mathrm{l})$} \\
\hline $\mathrm{HCO}_{3}^{-}$ & 0.06 & 0.09 & 0.10 \\
\hline $\mathrm{Cl}^{-}$ & 1.60 & 1.55 & 1.50 \\
\hline $\mathrm{SO}_{4}^{-2}$ & 0.93 & 0.84 & 0.74 \\
\hline \multicolumn{4}{|l|}{ Macronutrients (ppm) } \\
\hline $\mathrm{N}$ & 45.50 & 40.20 & 30.00 \\
\hline $\mathrm{P}$ & 9.20 & 8.50 & 6.00 \\
\hline $\mathrm{K}$ & 360 & 310 & 270 \\
\hline
\end{tabular}

\section{The following treatments conducted:-}

\section{1- Irrigation frequencies}

$\mathrm{I}_{1}$ - Every 21- days interval.

$\mathrm{I}_{2}$ - Every 28 - days interval.

$\mathrm{I}_{3}$ - Every 35- days interval.

\section{2- Vitamins soaking}

$\mathrm{V}_{0}$ - Control (soaking in water)

$\mathrm{V}_{1}$ - Soaking in $200 \mathrm{ppm}$ of ascorbic acid

$\mathrm{V}_{2}$ - Soaking in $200 \mathrm{ppm}$ of pyridoxine

\section{3- Potassium application}

$\mathrm{K}_{0}$ - Control (without $\mathrm{K}$ fertilizer)

$\mathrm{K}_{1}$ - All dose as a soil application

$\mathrm{K}_{2}$ - Half dose as a soil application and another foliar spray.

$\mathrm{K}_{3}$ - All dose as a foliar spray.

Wheat seeds were soaked in water, solution of ascorbic acid and pyridoxine for 6 hours, and then sown in the prepared soil on the 24 November for growing season. The three irrigation frequencies, every 21-, 28- and 35-days intervals which started after 35- days from planting and provided 6, 5, 4 irrigations per growth season. The required amount of irrigation water to each plot for each one irrigation was 
calculated to be $1.25 \mathrm{~m}^{3}$. This amount of irrigation water was conveyed to the plot through surface pipes from the main pipe in the sub- surface irrigation system. Also, potassium fertilizer was applied in the form of potassium sulphate $\left(48 \% \mathrm{~K}_{2} \mathrm{O}\right)$ at the rate $24 \mathrm{Kg} \mathrm{K}_{2} \mathrm{O} / \mathrm{Fed}$. Concerning soil application, it was divided into equal two doses; the first one was added with planting irrigation and the second one with the starting irrigation treatments. However, foliar application was sprayed two times with the first and the second irrigations of irrigation treatments, with the concentration of $3 \%$ of $\mathrm{K}_{2} \mathrm{O}$ solution. Regarding soil and foliar application treatment, soil application was given with planting irrigation and the foliar application was sprayed with the starting irrigation treatments. Other cultural practices were performed as usual.

At harvest, one square meter from each sub-sub- plot was sampled and the number of spikes $/ \mathrm{m}^{2}$ was estimated. Moreover, a random sample of ten plants was collected from the central area of each plot to estimate the following characters:-

1 - $\quad 1000$ - grains weight $(\mathrm{g})$.

2- $\quad$ Straw yield (ton/ Fed).

3- $\quad$ Grain yield (ard / Fed), where $\operatorname{ardab}=150 \mathrm{~kg}$.

4- $\quad$ Biological yield $(\mathrm{Kg})=$ Grain yield/fed + Straw yield $/$ fed.

5- Harvest index ( Hi) calculated as :

$$
\mathrm{Hi}=\frac{\text { Grain yield } / \text { Fed }}{\text { Grain yield }+ \text { Straw yield / Fed. }} \times 100
$$

Determination of $\mathbf{N}, \mathbf{P}$ and $\mathbf{K}$ were performed as described by Jackson (1970).Field water use efficiency was calculated for each treatment by dividing the yield $(\mathrm{Kg} / \mathrm{Fed})$ on unit of applied irrigation expressed as cubic meter of water (Al$\operatorname{Barrak}(2006)$.

Total amino acids were determined according the method of Moore and Stein (1948). The total free amino acids were calculated as $\mu \mathrm{g} \mathrm{mg}^{-1}$ dry weight / dry grain.

The obtained data obtained in each season were statistically analyzed according to producer outline by Gomez and Gomez (1984). Comparisons of means were estimated using the Least Significant Difference (L.S.D) at $5 \%$ level of probability. 


\section{Results And Discussion}

Wheat Yield and Its Components :

a- Effect of main parameters:

The data presented in Table (2) indicate that the grain yield and its components were significantly affected by irrigation frequencies. The irrigation every 21-day interval gave higher values of grain yield (17.72 and $17.08 \mathrm{ard} / \mathrm{Fed}$.), and all studied characters as compared with the other irrigation frequencies (28-day and 35- day intervals) in both 2005/2006 and 2006/2007 seasons. All studied other characters showed significant increases: 1000-grain weight (8.04 and 7.15\%), straw yield (17.33 and $22.30 \%$ ), grain yield (21.12and 20.96\%) and biological yield (18.73 and $21.74 \%$ ) when irrigated every 21-days interval compared with the irrigation every 35- days in both seasons, respectively. These finding are in harmony with those of Hussein (2005) and El-Saei et al. (2006).

It was also found that the grain yield and its components were significantly affected by vitamins soaking, except the harvest index of wheat during two seasons (Table,2). The soaking in pyridoxine $\left(\mathrm{V}_{2}\right)$ obtained higher values of grain yield (16.69 and $16.44 \mathrm{ard} / \mathrm{Fed}$.), and all other characters as compared with control $\left(\mathrm{V}_{0}\right)$ and ascorbic acid $\left(\mathrm{V}_{1}\right)$ in both seasons. These results indicated that significant increases in 1000-grain weight (2.65 and 3.18\%), straw yield (14.29 and 15.62\%), grain yield (15.34 and 16.64\%) and biological yield (14.61 and $15.97 \%$ ) when performance soaking in $\left(\mathrm{V}_{2}\right)$ as compared with control $\left(\mathrm{V}_{0}\right)$ during 2005/2006 and 2006/2007 seasons, respectively. These results are in agreement with those of Zidan (1990) who found that effects of some vitamins such as pyridoxine on growth parameters and some metabolic activities of wheat (Triticum aestivum L.) were comparatively raised.

Results in Table (2) reveal that the grain yield and its components were significantly affected by applied potassium application only except harvest index in both seasons. The superior grain yield of wheat per feddan which resulted from treatment all potassium as foliar spray (17.43 and $17.27 \mathrm{ard} / \mathrm{Fed}$.) during growing $2005 / 2006$ and 2006/2007 seasons, respectively. On the other hand, application of potassium all does as foliar spray gave significant increases of 1000-grain weight (4.87 and 4.67\%), straw yield (23.33 and $22.54 \%$ ), grain yield (23.17 and $32.51 \%$ ) and biological yield (23.37 and $23.51 \%$ ) as compared with control (without potassium fertilizer) in both seasons, respectively. Similar results were obtained by Saad et al. (1990), El-Defan et al. (1999), El-Kholy (2000), Mahdy and Teama (2000), Abd-Alla (2002), Abdel-Aziz et al., 2004 Hussien (2005) and El-Saei et al. (2006). 
IMPACT OF IRRIGATION FREQUENCIES, POTASSIUM...

Table (2): Effect main parameters on yield and its components of wheat during two seasons.

\begin{tabular}{|c|c|c|c|c|c|c|c|c|c|c|c|}
\hline \multirow[b]{2}{*}{ Parameters } & \multirow[t]{2}{*}{ Level } & \multicolumn{2}{|c|}{$\begin{array}{c}\text { 1000-grain weight } \\
\text { (g) }\end{array}$} & \multicolumn{2}{|c|}{$\begin{array}{c}\text { Straw yield } \\
\text { (ton/ Fed.) }\end{array}$} & \multicolumn{2}{|c|}{$\begin{array}{c}\text { Grain yield } \\
\text { (ard/Fed.) }\end{array}$} & \multicolumn{2}{|c|}{$\begin{array}{c}\text { Biological yield } \\
(\mathrm{kg} / \mathrm{Fed})\end{array}$} & \multicolumn{2}{|c|}{$\begin{array}{c}\text { Harvest index } \\
(\%)\end{array}$} \\
\hline & & $2005 / 2006$ & $2006 / 2007$ & $2005 / 2006$ & $2006 / 2007$ & $2005 / 2006$ & $2006 / 2007$ & $2005 / 2006$ & $2006 / 2007$ & $2005 / 2006$ & $2006 / 2007$ \\
\hline \multirow{3}{*}{$\begin{array}{l}\text { Irrigation } \\
\text { frequencies }\end{array}$} & $\mathbf{I}_{1}$ & 45.13 & 44.77 & 4.27 & 4.44 & 17.72 & 17.08 & 6888.65 & 6998.31 & 38.80 & 36.97 \\
\hline & $\mathbf{I}_{2}$ & 43.30 & 43.08 & 4.22 & 4.11 & 15.12 & 14.81 & 6492.77 & 6334.33 & 34.86 & 34.99 \\
\hline & $\mathbf{I}_{3}$ & 41.50 & 41.57 & 3.53 & 3.45 & 13.78 & 13.50 & 5598.86 & 5476.92 & 36.92 & 36.93 \\
\hline \multicolumn{2}{|c|}{ L.S.D at 0.05 level } & 0.26 & 0.43 & 0.41 & 0.17 & 0.52 & 0.19 & 447.55 & 190.76 & 2.42 & 0.62 \\
\hline \multirow{3}{*}{$\begin{array}{l}\text { Vitamins } \\
\text { soaking }\end{array}$} & $\mathrm{V}_{0}$ & 42.59 & 42.31 & 3.66 & 3.62 & 14.13 & 13.63 & 5782.68 & 5661.63 & 36.63 & 36.16 \\
\hline & $\mathrm{V}_{1}$ & 43.58 & 43.42 & 4.09 & 4.10 & 15.74 & 15.35 & 6414.53 & 6398.04 & 36.71 & 36.14 \\
\hline & $\mathrm{V}_{2}$ & 43.75 & 43.7 & 4.27 & 4.29 & 16.77 & 16.44 & 6783.07 & 6750.22 & 37.14 & 36.58 \\
\hline \multicolumn{2}{|c|}{\begin{tabular}{|l|} 
L.S.D at 0.05 level \\
\end{tabular}} & 0.47 & 0.5 & 0.15 & 0.17 & 0.48 & 0.11 & 186.22 & 219.13 & N.S & N.S \\
\hline \multirow{4}{*}{$\begin{array}{c}\text { Potassium } \\
\text { application }\end{array}$} & $\mathbf{K}_{0}$ & 42.23 & 42.08 & 3.45 & 3.47 & 13.66 & 13.21 & \begin{tabular}{|l|}
5444.37 \\
\end{tabular} & \begin{tabular}{|l|l|}
5448.33 \\
\end{tabular} & 37.29 & 36.41 \\
\hline & $K_{1}$ & 42.85 & 42.85 & 3.81 & 3.82 & 14.77 & 14.34 & 6026.50 & 5968.17 & 36.83 & 36.10 \\
\hline & $\mathbf{K}_{2}$ & 43.76 & 43.49 & 4.27 & 4.23 & 16.30 & 15.63 & 6716.82 & 6576.69 & 36.49 & 35.75 \\
\hline & $\mathbf{K}_{3}$ & 44.39 & 44.14 & 4.50 & 4.48 & 17.43 & 17.15 & \begin{tabular}{|l|l|}
7119.35 \\
\end{tabular} & \begin{tabular}{|l|l|}
7086.22 \\
\end{tabular} & 36.83 & 36.92 \\
\hline \multicolumn{2}{|c|}{ L.S.D at 0.05 level } & $\begin{array}{l}0.37 \\
\end{array}$ & 0.43 & 0.17 & 0.15 & 0.56 & 0.17 & 199.37 & 154.28 & N.S & N.S \\
\hline
\end{tabular}

$b$ - Effects of irrigation frequencies with both vitamins soaking and potassium application:

Results given in Table (3) indicate that the wheat grain yield and its components of wheat were significantly affected only except 1000-grain weight and harvest index. The interaction effects between irrigation every 21-days and vitamin soaking in pyridoxine gave higher values of grain yield of wheat (19.09 and $18.62 \mathrm{ard} / \mathrm{fed}$.) and all other characters only except harvest index as compared with the other treatments in both seasons, respectively. The interaction effect between irrigation every 21 - day and soaking in pyridoxine gave significant increases of : 1000-grain weight (7.72 and $10.52 \%$ ), straw yield (28.84 and $34.28 \%$ ), grain yield (34.00 and $34.64 \%)$ and biological yield (30.78 and $34.40 \%$ ) as compared with irrigation every 35 -days and soaking in water during growing seasons (2005/2006 and 2006/2007), respectively.

Data in Table (3) show the interaction between irrigation frequencies and potassium application which had significant effects on the straw yield, biological yield and harvest index in both seasons. The highest value of 1000-grain weight (46.40 and 46.03 gm.), straw yield (5.00 and 5.12 ton/fed.), grain yield (19.39 and $18.96 \mathrm{ard} / \mathrm{Fed}$.) and biological yield (7904.67 and $7967.83 \mathrm{~kg} / \mathrm{Fed}$ ) could be obtained when irrigation was applied every 21 -days and application of potassium as foliar spray in both seasons, respectively. These results are similar to those reported by Kansal and Sokhon (1973), Mohamed and Ali (1982), Hussein (2005) and ElSaei et al. (2006). 
Table (3): Effects of interaction between irrigation frequency and both soaking in vitamins and potassium application on yield and its components of wheat during two seasons.

\begin{tabular}{|c|c|c|c|c|c|c|c|c|c|c|c|}
\hline \multirow{2}{*}{$\begin{array}{l}\text { Irrigation } \\
\text { frequencies }\end{array}$} & \multirow{2}{*}{$\begin{array}{c}\text { Soaking in } \\
\text { vitamins }\end{array}$} & \multirow[b]{2}{*}{$\begin{array}{l}2005 / \\
2006\end{array}$} & \multirow[b]{2}{*}{\begin{tabular}{|l|}
2006 \\
$/ 2007$
\end{tabular}} & \multicolumn{2}{|c|}{$\begin{array}{c}\text { Straw yield } \\
\text { (ton/Fed.) }\end{array}$} & \multicolumn{2}{|c|}{$\begin{array}{l}\text { Grain yield } \\
\text { (ard/Fed.) }\end{array}$} & \multicolumn{2}{|c|}{$\begin{array}{c}\text { Biological yield } \\
\text { (kg/ Fed) }\end{array}$} & \multicolumn{2}{|c|}{$\begin{array}{c}\text { Harvest index } \\
(\%)\end{array}$} \\
\hline & & & & $\begin{array}{l}2005 / \\
2006\end{array}$ & $\begin{array}{l}2006 / \\
2007\end{array}$ & $\begin{array}{l}2005 / \\
2006\end{array}$ & $\begin{array}{l}2006 / \\
2007\end{array}$ & $\begin{array}{l}2005 / \\
2006\end{array}$ & $\begin{array}{l}2006 / \\
2007\end{array}$ & $\begin{array}{l}2005 / \\
2006\end{array}$ & $\begin{array}{l}2006 / \\
2007\end{array}$ \\
\hline \multirow{3}{*}{$I_{1}$} & $V_{0}$ & 44.18 & 43.67 & 3.67 & 3.79 & 16.36 & 15.41 & 6128.04 & 6104.38 & 40.27 & 38.23 \\
\hline & $V_{1}$ & 45.33 & 44.74 & 4.38 & 4.59 & 17.72 & 17.21 & \begin{tabular}{|l|}
6930.04 \\
\end{tabular} & \begin{tabular}{|l|}
7168.25 \\
\end{tabular} & 38.14 & 36.36 \\
\hline & $\mathrm{V}_{2}$ & 45.87 & 45.91 & 4.75 & 4.93 & 19.09 & 18.62 & \begin{tabular}{|l|}
7607.88 \\
\end{tabular} & \begin{tabular}{|l|}
7722.29 \\
\end{tabular} & 37.98 & 36.32 \\
\hline \multirow{3}{*}{$\mathbf{I}_{2}$} & $\mathrm{~V}_{0}$ & 42.83 & 42.17 & 3.94 & 3.82 & 13.42 & 13.30 & 5953.71 & 5815.00 & 33.71 & 34.24 \\
\hline & $V_{1}$ & 43.68 & 43.69 & 4.28 & 4.15 & 15.63 & 15.14 & 6624.38 & 6420.38 & 35.41 & 35.31 \\
\hline & $\mathbf{V}_{2}$ & 43.38 & 43.38 & \begin{tabular}{|l|}
4.45 \\
\end{tabular} & 4.36 & 16.36 & 16.07 & \begin{tabular}{|l|}
6900.21 \\
\end{tabular} & \begin{tabular}{|l|}
6767.63 \\
\end{tabular} & \begin{tabular}{|l|}
35.47 \\
\end{tabular} & 35.42 \\
\hline \multirow{3}{*}{$\mathbf{I}_{3}$} & $\mathrm{~V}_{0}$ & 40.77 & 41.08 & 3.38 & 3.24 & 12.60 & 12.17 & 5266.29 & 5065.50 & 35.91 & 36.01 \\
\hline & $V_{1}$ & 41.73 & 41.82 & 3.61 & 3.55 & 13.87 & 13.72 & 5689.17 & 5605.50 & 36.58 & 36.76 \\
\hline & $\mathbf{V}_{2}$ & 42.00 & 41.81 & 3.61 & 3.57 & 14.87 & 14.62 & 5841.13 & 5759.75 & 38.27 & 38.01 \\
\hline \multicolumn{2}{|c|}{\begin{tabular}{|l|} 
LSD at 0.05 level \\
\end{tabular}} & N.S & N.S & 0.26 & 0.30 & 0.82 & 0.21 & 322.54 & 297.65 & 1.69 & N.S \\
\hline $\begin{array}{l}\text { Irrigation } \\
\text { frequencies }\end{array}$ & $\begin{array}{l}\text { Potassium } \\
\text { application }\end{array}$ & & & & & & & & & & \\
\hline \multirow{4}{*}{$\mathbf{I}_{1}$} & $K_{0}$ & 43.79 & 43.27 & 3.30 & 3.60 & 15.75 & 15.73 & 5509.33 & 5960.00 & 41.76 & 39.99 \\
\hline & $\mathrm{K}_{1}$ & 44.63 & 44.60 & 3.86 & 4.13 & 17.29 & 16.21 & 6456.83 & 6561.50 & 40.38 & 37.26 \\
\hline & $\mathbf{K}_{2}$ & 45.69 & 45.19 & 4.91 & 4.89 & 18.46 & 17.41 & 7683.78 & 7503.89 & 36.09 & 34.86 \\
\hline & $\mathbf{K}_{3}$ & 46.40 & 46.03 & 5.00 & 5.12 & 19.39 & 18.96 & \begin{tabular}{|l|}
7904.67 \\
\end{tabular} & \begin{tabular}{|l|}
7967.83 \\
\end{tabular} & 36.96 & 35.77 \\
\hline \multirow{4}{*}{$\mathbf{I}_{2}$} & $\mathbf{K}_{\mathbf{0}}$ & 42.10 & 42.44 & 3.81 & 3.67 & 13.02 & 12.06 & 5767.61 & 5479.50 & 33.85 & 33.04 \\
\hline & $\mathrm{K}_{1}$ & 42.92 & 42.64 & 4.12 & 3.99 & 13.99 & 14.11 & 6214.67 & 6103.33 & 33.82 & 34.71 \\
\hline & $\mathbf{K}_{2}$ & 43.58 & 43.18 & 4.37 & 4.26 & 15.98 & 15.36 & \begin{tabular}{|l|}
6761.89 \\
\end{tabular} & \begin{tabular}{|l|}
6567.83 \\
\end{tabular} & 35.38 & 35.07 \\
\hline & $\mathbf{K}_{3}$ & 44.58 & 44.06 & 4.60 & 4.52 & 17.48 & 17.72 & 7226.89 & 7186.67 & 36.39 & 37.13 \\
\hline \multirow{4}{*}{$\mathbf{I}_{3}$} & $\mathbf{K}_{\mathbf{0}}$ & 40.80 & 40.53 & 3.22 & 3.13 & 12.22 & 11.84 & 5056.17 & 4905.50 & 36.25 & 36.19 \\
\hline & $\mathbf{K}_{1}$ & 40.99 & 41.30 & 3.45 & 3.34 & 13.03 & 12.69 & 5408.00 & 5239.67 & 36.30 & 36.33 \\
\hline & $\mathbf{K}_{2}$ & 42.02 & 42.11 & 3.54 & 3.54 & 14.45 & 14.10 & 5704.78 & 5658.33 & 37.99 & 37.33 \\
\hline & $\mathbf{K}_{3}$ & 42.18 & 42.34 & 3.91 & 3.80 & 15.42 & 15.38 & 6226.50 & 6104.17 & 37.13 & 37.86 \\
\hline \multicolumn{2}{|c|}{ LSD at 0.05 level } & N.S & N.S & 0.30 & 0.26 & N.S & 0.30 & 345.32 & 267.22 & 2.16 & 1.77 \\
\hline
\end{tabular}

$c$ - Effect of interaction between irrigation frequencies, soaking in vitamins and potassium application:

Results in Table (4) indicate that the interaction between irrigation frequencies, soaking in vitamins and potassium application had significant effect on grain yield only in 2006/2007 season. While, the 1000-grain weight, straw yield, grain yield, biological yield and harvest index in both seasons were non significant, respectively. The highest values of 1000-grain weight (47.42 and $47.17 \mathrm{gm}$ ), straw yield (5.62 and 5.71 ton/Fed.), grain yield (21.26 and $21.12 \mathrm{ard} / \mathrm{fed}$.) and biological yield ( 8878.17 and $8868.00 \mathrm{~kg} / \mathrm{Fed}$ ) were obtained by irrigating t every 21 - days, soaking pyridoxine and application of all potassium fertilizer as foliar spray in both seasons, respectively (Table 4 ).

However, the lowest 1000-grain weight (40.13 and $40.12 \mathrm{gm}$.), straw yield (3.12 and 3.05 ton/Fed.), grain yield (11.95 and 11.19 ard/Fed.) and biological yield (4912.00 and $4728.50 \mathrm{~kg}$ ) were given when the plants were irrigated every 35-days, 
soaking in water and without potassium fertilizer in 2005/2006 and 2006/2007 seasons, respectively (Table 4).

These results are in agreement with those obtained by El-Kholy (2000),Hussien (2005) and El-Saei et al. (2006).

Table (4): Effect of interaction between irrigation frequency, soaking in vitamins and potassium application on yield and its components of wheat during two seasons.

\begin{tabular}{|c|c|c|c|c|c|c|c|c|c|c|c|c|}
\hline \multirow{2}{*}{$\begin{array}{l}\text { Irrigation } \\
\text { frequency }\end{array}$} & \multirow{2}{*}{$\begin{array}{c}\text { Soaking } \\
\text { in } \\
\text { vitamins }\end{array}$} & \multirow{2}{*}{$\begin{array}{l}\text { Potassium } \\
\text { application }\end{array}$} & \multicolumn{2}{|c|}{$\begin{array}{l}\text { 1000-grain } \\
\text { weight }(\mathrm{g})\end{array}$} & \multicolumn{2}{|c|}{$\begin{array}{l}\text { Straw yield } \\
\text { (ton/Fed.) }\end{array}$} & \multicolumn{2}{|c|}{$\begin{array}{l}\text { Grain yield } \\
(\operatorname{ard} / \text { Fed.) }\end{array}$} & \multicolumn{2}{|c|}{$\begin{array}{c}\begin{array}{c}\text { Biological yield } \\
(\mathrm{kg} / \mathrm{Fed})\end{array} \\
\end{array}$} & \multicolumn{2}{|c|}{$\begin{array}{c}\text { Harvest index } \\
(\%)\end{array}$} \\
\hline & & & \begin{tabular}{|l}
$2005 /$ \\
2006 \\
\end{tabular} & \begin{tabular}{|l|}
$2006 /$ \\
2007 \\
\end{tabular} & \begin{tabular}{|l}
$2005 /$ \\
2006 \\
\end{tabular} & $\begin{array}{l}2006 / \\
2007\end{array}$ & \begin{tabular}{|l}
$2005 /$ \\
2006 \\
\end{tabular} & \begin{tabular}{|l}
$2006 /$ \\
2007 \\
\end{tabular} & $\begin{array}{l}2005 / \\
2006\end{array}$ & $\begin{array}{l}2006 / \\
2007\end{array}$ & \begin{tabular}{|l}
$2005 /$ \\
2006 \\
\end{tabular} & $\begin{array}{l}2006 / \\
2007\end{array}$ \\
\hline \multirow{12}{*}{$\mathrm{I}_{1}$} & \multirow{4}{*}{$\mathrm{V}_{0}$} & $\mathrm{~K}_{0}$ & 42.32 & 42.15 & 3.11 & 3.21 & 14.63 & 14.41 & 5301.17 & 5371.50 & 41.37 & 40.90 \\
\hline & & $\mathrm{K}_{1}$ & 43.97 & 43.60 & 3.21 & 3.35 & 15.74 & 14.67 & 5570.50 & 5550.50 & 42.55 & 39.64 \\
\hline & & $\mathrm{K}_{2}$ & 45.02 & 44.21 & 4.18 & 4.21 & 16.36 & 15.21 & 6636.83 & \begin{tabular}{|l|l|}
6491.50 \\
\end{tabular} & \begin{tabular}{|l|}
36.95 \\
\end{tabular} & 35.17 \\
\hline & & $\mathrm{K}_{3}$ & 45.59 & 44.72 & 4.20 & 4.40 & 18.71 & \begin{tabular}{|l|}
17.36 \\
\end{tabular} & 7003.67 & \begin{tabular}{|l|}
7004.00 \\
\end{tabular} & 40.20 & 37.19 \\
\hline & \multirow{4}{*}{$\mathrm{V}_{1}$} & $\mathrm{~K}_{0}$ & 44.61 & 43.51 & 3.35 & 3.47 & 16.44 & 16.25 & \begin{tabular}{|l|l|}
5369.33 \\
\end{tabular} & \begin{tabular}{|l|}
5907.50 \\
\end{tabular} & 42.40 & 41.48 \\
\hline & & $\mathrm{K}_{\mathrm{I}}$ & 44.82 & 44.25 & 3.92 & 4.67 & 17.56 & 16.61 & 6553.50 & \begin{tabular}{|l|l}
7161.50 \\
\end{tabular} & 40.16 & 34.79 \\
\hline & & $\mathrm{K}_{2}$ & 45.73 & 45.00 & 5.09 & 4.95 & 18.71 & 17.55 & \begin{tabular}{|l|}
7893.17 \\
\end{tabular} & 7582.50 & 35.54 & 34.72 \\
\hline & & $\mathrm{K}_{3}$ & 46.18 & 46.21 & 5.18 & 5.26 & 18.18 & 18.41 & 7904.17 & 8021.50 & 34.46 & 34.45 \\
\hline & \multirow[t]{4}{*}{$\mathrm{V}_{2}$} & $\mathrm{~K}_{0}$ & 44.45 & 44.15 & 3.34 & 4.12 & 16.18 & 16.54 & \begin{tabular}{|l|}
5857.50 \\
\end{tabular} & \begin{tabular}{|l|}
6601.00 \\
\end{tabular} & 41.51 & 37.59 \\
\hline & & $\mathrm{K}_{1}$ & 45.29 & 45.95 & 4.46 & 4.37 & 18.58 & 17.35 & 7246.50 & 6972.50 & 38.44 & 37.36 \\
\hline & & $\mathrm{K}_{2}$ & 46.32 & 46.35 & 5.47 & 5.52 & 20.32 & \begin{tabular}{|l}
19.47 \\
\end{tabular} & 8521.33 & \begin{tabular}{|l}
8437.67 \\
\end{tabular} & 35.76 & 34.68 \\
\hline & & $\mathrm{K}_{3}$ & 47.42 & 47.17 & 5.62 & 5.71 & 21.26 & 21.12 & 8806.17 & \begin{tabular}{|l|}
8878.00 \\
\end{tabular} & 36.22 & 35.68 \\
\hline \multirow{12}{*}{$\mathrm{I}_{2}$} & \multirow{4}{*}{$\mathrm{V}_{0}$} & $\mathrm{~K}_{0}$ & 41.18 & 41.20 & 3.60 & 3.49 & 11.64 & 11.45 & \begin{tabular}{|l|}
5345.50 \\
\end{tabular} & \begin{tabular}{|l|}
5207.50 \\
\end{tabular} & 32.60 & 32.98 \\
\hline & & $\mathrm{K}_{1}$ & 42.68 & 41.79 & 3.86 & 3.67 & 12.62 & 12.19 & 5756.33 & 5498.50 & 32.84 & 33.26 \\
\hline & & $\mathrm{K}_{2}$ & 43.61 & 42.52 & 4.10 & 3.95 & 14.22 & \begin{tabular}{|l}
13.75 \\
\end{tabular} & 6232.50 & \begin{tabular}{|l}
6012.50 \\
\end{tabular} & 34.22 & 34.39 \\
\hline & & $\mathrm{K}_{3}$ & 43.84 & 43.15 & 4.20 & 4.17 & 15.20 & \begin{tabular}{|l|}
15.81 \\
\end{tabular} & 6480.50 & 6541.50 & \begin{tabular}{|l|}
35.17 \\
\end{tabular} & 36.32 \\
\hline & \multirow{4}{*}{$\mathrm{V}_{1}$} & $\mathrm{~K}_{0}$ & 42.92 & 43.67 & 3.89 & 3.71 & 13.83 & 12.41 & \begin{tabular}{|l|}
5967.33 \\
\end{tabular} & \begin{tabular}{|l|}
5571.50 \\
\end{tabular} & \begin{tabular}{|l|}
34.79 \\
\end{tabular} & 33.43 \\
\hline & & $\mathrm{K}_{1}$ & 43.48 & 43.21 & 4.04 & 3.92 & 14.35 & 14.59 & 6192.50 & \begin{tabular}{|l|}
6109.00 \\
\end{tabular} & 35.03 & 36.08 \\
\hline & & $\mathrm{K}_{2}$ & 34.79 & 43.51 & 4.43 & 4.32 & 16.09 & 15.22 & \begin{tabular}{|l}
6843.50 \\
\end{tabular} & \begin{tabular}{|l|}
6603.00 \\
\end{tabular} & 35.26 & 34.58 \\
\hline & & $\mathrm{K}_{3}$ & 44.54 & 44.37 & 4.76 & 4.65 & 18.25 & 18.32 & \begin{tabular}{|l|}
7494.17 \\
\end{tabular} & \begin{tabular}{|l|}
7398.00 \\
\end{tabular} & \begin{tabular}{|l|}
36.56 \\
\end{tabular} & 37.15 \\
\hline & \multirow[t]{4}{*}{$\mathrm{V}_{2}$} & $\mathrm{~K}_{0}$ & 42.20 & 42.45 & 3.95 & 3.81 & 13.60 & 12.33 & \begin{tabular}{|l|}
5990.00 \\
\end{tabular} & \begin{tabular}{|l|}
5659.50 \\
\end{tabular} & \begin{tabular}{|l|}
34.17 \\
\end{tabular} & 32.71 \\
\hline & & $\mathrm{K}_{1}$ & 42.61 & 42.91 & 4.45 & 4.37 & 14.99 & 15.55 & 6695.17 & 6702.50 & \begin{tabular}{|l|}
33.59 \\
\end{tabular} & 34.81 \\
\hline & & $\mathrm{K}_{2}$ & 43.34 & \begin{tabular}{|l|}
43.52 \\
\end{tabular} & 4.57 & 4.52 & 17.62 & \begin{tabular}{|l|}
17.12 \\
\end{tabular} & \begin{tabular}{|l|}
7209.67 \\
\end{tabular} & \begin{tabular}{|l|}
7088.00 \\
\end{tabular} & \begin{tabular}{|l|}
36.66 \\
\end{tabular} & 36.23 \\
\hline & & $\mathrm{K}_{3}$ & 45.37 & 44.65 & 4.84 & 4.73 & 19.24 & 19.27 & 7706.00 & \begin{tabular}{|l|}
7620.50 \\
\end{tabular} & 37.45 & 37.93 \\
\hline \multirow[t]{12}{*}{$\mathrm{I}_{3}$} & \multirow{4}{*}{$\mathrm{V}_{0}$} & $\mathrm{~K}_{0}$ & 40.13 & 40.12 & 3.12 & 3.05 & 11.95 & 11.19 & \begin{tabular}{|l|}
4912.00 \\
\end{tabular} & \begin{tabular}{|l|l|}
4728.50 \\
\end{tabular} & \begin{tabular}{|l|}
36.49 \\
\end{tabular} & 35.50 \\
\hline & & $\mathrm{K}_{1}$ & 40.30 & 40.45 & 3.24 & 3.11 & 12.01 & 11.42 & \begin{tabular}{|l|}
5042.00 \\
\end{tabular} & 4823.00 & 35.74 & 35.52 \\
\hline & & $\mathrm{K}_{2}$ & 41.23 & 41.83 & 3.42 & 3.23 & 12.32 & 12.35 & 5257.33 & \begin{tabular}{|l}
5082.50 \\
\end{tabular} & \begin{tabular}{|l|}
35.09 \\
\end{tabular} & 36.45 \\
\hline & & $\mathrm{K}_{3}$ & 41.43 & 41.92 & 3.72 & 3.57 & 14.20 & \begin{tabular}{|l|}
13.72 \\
\end{tabular} & \begin{tabular}{|l|}
5853.83 \\
\end{tabular} & \begin{tabular}{|l|}
5628.00 \\
\end{tabular} & \begin{tabular}{|l|}
36.31 \\
\end{tabular} & 36.59 \\
\hline & \multirow{4}{*}{$\mathrm{V}_{1}$} & $\mathrm{~K}_{0}$ & 41.09 & 40.10 & 3.25 & 3.13 & 12.26 & 12.11 & \begin{tabular}{|l|}
5088.50 \\
\end{tabular} & \begin{tabular}{|l|}
4946.50 \\
\end{tabular} & 36.11 & 36.73 \\
\hline & & $\mathrm{K}_{1}$ & 41.05 & 42.00 & 3.65 & 3.35 & 12.70 & 12.45 & 5555.00 & 5217.50 & 34.45 & 35.81 \\
\hline & & $\mathrm{K}_{2}$ & 42.24 & 42.32 & 3.48 & 3.71 & 15.19 & 14.50 & \begin{tabular}{|l|}
5758.50 \\
\end{tabular} & \begin{tabular}{|l}
5885.00 \\
\end{tabular} & \begin{tabular}{|l|}
39.59 \\
\end{tabular} & 36.96 \\
\hline & & $\mathrm{K}_{3}$ & 42.52 & 42.85 & 4.06 & 4.00 & 15.32 & \begin{tabular}{|l}
15.82 \\
\end{tabular} & 6354.67 & \begin{tabular}{|l}
6373.00 \\
\end{tabular} & 36.18 & 37.53 \\
\hline & \multirow[t]{4}{*}{$\mathrm{V}_{2}$} & $\mathrm{~K}_{0}$ & 41.19 & 41.36 & 3.30 & 3.21 & 12.45 & 12.21 & 5168.00 & 5041.50 & 36.15 & 36.34 \\
\hline & & $\mathrm{K}_{1}$ & 41.63 & 41.45 & 3.47 & 3.55 & 14.38 & \begin{tabular}{|l|}
14.19 \\
\end{tabular} & \begin{tabular}{|l|}
5627.00 \\
\end{tabular} & \begin{tabular}{|l|}
5678.50 \\
\end{tabular} & \begin{tabular}{|l|}
38.71 \\
\end{tabular} & 37.65 \\
\hline & & $\mathrm{K}_{2}$ & 42.59 & 42.17 & 3.71 & 3.69 & 15.92 & \begin{tabular}{|l}
15.45 \\
\end{tabular} & 6098.50 & \begin{tabular}{|l}
6007.50 \\
\end{tabular} & 39.30 & 38.57 \\
\hline & & $\mathrm{K}_{3}$ & 42.59 & 42.25 & 3.96 & 3.82 & 16.74 & 16.61 & 6471.00 & 6311.50 & 38.90 & 39.47 \\
\hline \multicolumn{3}{|c|}{ L.S.D. at 0.05 level } & N.S. & N.S. & N.S. & N.S. & N.S. & $0 . .51$ & N.S. & N.S. & N.S. & N.S. \\
\hline
\end{tabular}

\section{Macronutrients content in grains:}

Data in Table (5) show that the macronutrients content of wheat grains is significantly affected by using different treatments. During the two seasons, 28-days irrigation interval significantly increased the nitrogen and phosphorus contents, 
whereas, the highest value of potassium content (0.36 and 0.35) could be detected when the plants were subjected to irrigation at 21- days interval. . Soaking grains in pyridoxine gave the highest values of nitrogen (2.45) and phosphorus (0.21) in both seasons. While the soaking in water gave the highest values of potassium content (0.38 and 0.37) as compared with any vitamin in both seasons, respectively. Potassium application as foliar spray increased the contents of macronutrient in wheat grains. In general, the macronutrients content increased due to potassium application as soil addition toward foliar spray treatment in growing seasons.

The effects of irrigation interval $\mathrm{x}$ soaking in vitamins and irrigation interval $\mathrm{x}$ potassium application interactions on macronutrients content were significantly increased, with the highest values of macronutrients were observed when plants irrigated at 28-day interval (Table 6).

The interaction of irrigation interval $\mathrm{x}$ vitamin $\mathrm{x}$ potassium application significantly affected the macronutrient contents in wheat grains (Table 7). Results clearly show that irrigation at 28-days interval with soaking in ascorbic acid and potassium application as foliar spray realized the highest values of nitrogen and phosphorus contents (2.96 and 0.25) in wheat grains. However, the lower values were could be detected under irrigating the plants at 21 day interval with soaking in water and without potassium fertilizer (Table 7). Also, the highest amounts of potassium content $(0.45)$ in wheat grains was found under irrigation at 28-days interval with foliar spray of potassium fertilizer but without soaking in any vitamins (soaking in water), however, the lower values were recorded by soaking in both vitamins.

These results are in agreement with those obtained by Ibrahim et al. (1986) and El-Sayed (2007), they concluded that the nitrogen percentage in wheat grains was increased with the moderate soil moisture contents.

\section{Total amino acids content in grains:}

Data in Tables (5, 6 and 7) indicate that total amino acids contents were significantly affected by the irrigation intervals, soaking in vitamins and potassium application as well as their combinations. It is obvious from these data that, the content of total amino acids in both growing seasons increased with increasing irrigation interval up to 28-days. The maximum content of total amino acid (14.90 $\mu \mathrm{g} \mathrm{mg}^{-1}$ dry weight) was obtained in plants irrigated at 28-days interval with soaking in ascorbic acid and foliar sprayed with potassium fertilizer, while its minimum content ( $7.49 \mu \mathrm{g} \mathrm{mg}^{-1}$ dry weight) was recorded under irrigation at 35-days interval 
combined with soaking in ascorbic acid and without potassium application. Using pyridoxine vitamin increased the total amino acid contents, whereas, pyridoxine $\left(\mathrm{B}_{6}\right.$ vitamin) is the pyridine ring containing precursor of essential enzyme pyridoxal phosphate, which is utilized by enzymes in all phases of amino acid metabolism (Bender, 1985).Also, foliar spray of potassium fertilizer increased the total amino acid contents in both seasons, whereas potassium is essential in protein synthesis (Marschner 1995).

\section{Field water use efficiency:}

It is the weight of marketable crop production per the volume unit of applied irrigation was expressed as cubic meter of water. Data listed in Table (8) and illustrated in Fig.(1) revealed that the values of field water use efficiency were affected by water stress, where it was increased by increasing interval between irrigation. It could be concluded that in both seasons the field water use efficiency increased when moisture stress increased. The highest value of field water use efficiency was calculated when plant irrigated at 35-days interval with soaking in pyridoxine and foliar spray of potassium fertilizer. These results are in accordance with those reported by Hussien et al. (2000), El-Saei et al. (2006) and El-Sayed (2007).

Table (5) : Effect of different treatments on micronutrients ( N, P, K) and total amino acid contents ( $\mu \mathrm{g} \mathrm{mg}^{-1}$ dry weight ) of wheat grains during two seasons.

\begin{tabular}{|c|c|c|c|c|c|c|c|c|c|}
\hline $\begin{array}{c}\text { Main } \\
\text { Parameters }\end{array}$ & Level & \multicolumn{2}{|c|}{$\mathbf{N} \%$} & \multicolumn{2}{|c|}{$\mathbf{P} \%$} & \multicolumn{2}{|c|}{$\mathbf{K} \%$} & \multicolumn{2}{|c|}{$\begin{array}{l}\text { Total Amino } \\
\text { Acids }\end{array}$} \\
\hline \multirow{4}{*}{$\begin{array}{c}\text { Irrigation } \\
\text { interval }\end{array}$} & & $\begin{array}{l}2005 / \\
2006\end{array}$ & $\begin{array}{l}2006 / \\
2007\end{array}$ & $\begin{array}{l}2005 / \\
2006\end{array}$ & $\begin{array}{l}2006 / \\
2007\end{array}$ & $\begin{array}{l}2005 / \\
2006\end{array}$ & $\begin{array}{l}2006 / \\
2007\end{array}$ & $\begin{array}{l}2005 / \\
2006\end{array}$ & $\begin{array}{l}2006 / \\
2007\end{array}$ \\
\hline & $\mathrm{I}_{1}$ & 2.35 & 2.32 & 0.181 & 018 & 0.36 & 0.352 & 12.23 & 11.11 \\
\hline & $\mathrm{I}_{2}$ & 2.55 & 2.41 & 0.21 & 0.21 & 0.33 & 0.33 & 12.85 & 11.79 \\
\hline & $\mathrm{I}_{3}$ & 2.32 & 2.32 & 0.18 & 0.18 & 0.34 & 0.34 & 9.88 & 8.61 \\
\hline \multicolumn{2}{|c|}{ L.S.D at $5 \%$} & 0.020 & 0.001 & 0.001 & 0.010 & 0.001 & 0.0001 & 0.040 & 0.070 \\
\hline \multirow{3}{*}{$\begin{array}{l}\text { Vitamins } \\
\text { Soaking }\end{array}$} & $\mathrm{V}_{0}$ & 2.32 & 2.25 & 0.170 & 0.171 & 0.38 & 0.37 & 11.04 & 9.80 \\
\hline & $\mathrm{V}_{1}$ & 2.44 & 2.35 & 0.20 & 0.20 & 0.33 & 0.32 & 11.70 & 10.70 \\
\hline & $\mathrm{V}_{2}$ & 2.45 & 2.45 & 0.198 & 0.199 & 0.322 & 0.33 & 12.32 & 11.01 \\
\hline \multicolumn{2}{|c|}{ L.S.D at $5 \%$} & 0.09 & 0.001 & 0.001 & 0.001 & 0.001 & 0.001 & 0.020 & 0.040 \\
\hline \multirow{4}{*}{$\begin{array}{l}\text { Potassium } \\
\text { application }\end{array}$} & $\mathrm{K}_{0}$ & 2.08 & 2.08 & 0.16 & 0.16 & 0.248 & 0.29 & 10.94 & 9.75 \\
\hline & $\mathrm{K}_{1}$ & 2.50 & 2.42 & 0.19 & 0.19 & 0.347 & 0.34 & 11.52 & 10.53 \\
\hline & $\mathrm{K}_{2}$ & 2.55 & 2.44 & 0.20 & 0.20 & 0.356 & 0.36 & 11.97 & 10.84 \\
\hline & $\mathrm{K}_{3}$ & 2.50 & 2.47 & 0.21 & 0.21 & 0.372 & 0.37 & 12.19 & 10.90 \\
\hline \multicolumn{2}{|c|}{ L.S.D at $5 \%$} & 0.01 & 0.01 & 0.001 & 0.001 & 0.01 & 0.01 & 0.04 & 0.05 \\
\hline
\end{tabular}


Table (6): Effect the interactions between, irrigation intervals, soaking in vitamins, potassium applications and irrigation intervals on micronutrients $(\mathrm{N}, \mathrm{P}, \mathrm{K})$ and total amino acid contents $\left(\mu \mathrm{g} \mathrm{mg}^{-1} \mathrm{dry}\right)$ weight of wheat grains during two seasons .

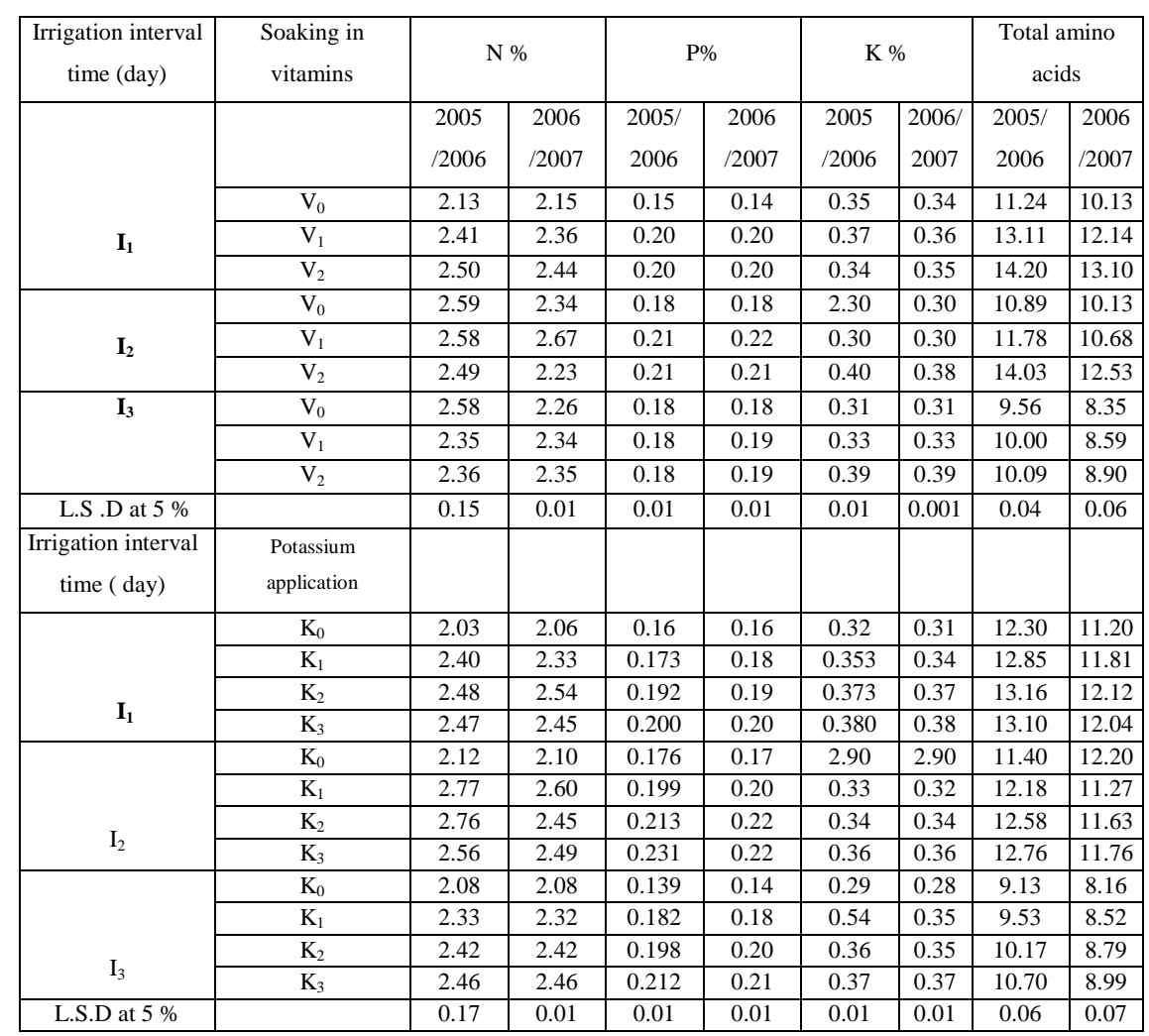


IMPACT OF IRRIGATION FREQUENCIES, POTASSIUM...

Table (7): Effect of interaction between irrigation interval, soaking in vitamins and potassium application on micronutrients $(N, P, K)$ and total amino acid contents ( $\mu \mathrm{g} \mathrm{mg}^{-1}$ dry weight ) of wheat grains during two seasons.

\begin{tabular}{|c|c|c|c|c|c|c|c|c|c|c|}
\hline $\begin{array}{l}\text { Irrigation } \\
\text { Interval }\end{array}$ & $\begin{array}{c}\text { Soaking } \\
\text { in }\end{array}$ & $\begin{array}{l}\text { Potassium } \\
\text { applications }\end{array}$ & \multicolumn{2}{|c|}{$\mathrm{N} \%$} & \multicolumn{2}{|c|}{$\mathrm{P} \%$} & \multicolumn{2}{|c|}{$\mathrm{K} \%$} & \multicolumn{2}{|c|}{ Total amino acids } \\
\hline \multirow{13}{*}{$\mathrm{I}_{1}$} & \multirow{5}{*}{$\mathrm{V}_{0}$} & & $\begin{array}{l}2005 / \\
2006\end{array}$ & $\begin{array}{l}2006 / \\
2007\end{array}$ & $\begin{array}{l}2005 / \\
2006\end{array}$ & $\begin{array}{l}2006 / \\
2007\end{array}$ & $\begin{array}{l}2005 / \\
2006\end{array}$ & $\begin{array}{l}2006 / \\
2007\end{array}$ & $\begin{array}{l}2005 / \\
2006\end{array}$ & $\begin{array}{l}2006 / \\
2007\end{array}$ \\
\hline & & $\mathrm{K}_{0}$ & 1.95 & 1.90 & 0.12 & 0.12 & 0.29 & 0.28 & 10.90 & 9.60 \\
\hline & & $\mathrm{K}_{1}$ & 2.10 & 2.05 & 0.13 & 0.14 & 0.30 & 0.30 & 10.95 & 9.730 \\
\hline & & $\mathrm{K}_{2}$ & 2.20 & 2.31 & 0.16 & 0.15 & 0.40 & 0.41 & 11.52 & 10.50 \\
\hline & & $\mathrm{K}_{3}$ & 2.25 & 2.32 & 0.17 & 0.16 & 0.42 & 0.41 & 11.60 & 10.70 \\
\hline & \multirow{4}{*}{$\mathrm{V}_{1}$} & $\mathrm{~K}_{0}$ & 2.0 & 1.96 & 0.18 & 0.18 & 0.34 & 0.33 & 12.80 & 11.60 \\
\hline & & $\mathrm{K}_{1}$ & 2.50 & 2.45 & 0.19 & 0.20 & 0.38 & 0.36 & 13.20 & 12.40 \\
\hline & & $\mathrm{K}_{2}$ & 2.60 & 2.52 & 0.21 & 0.21 & 0.38 & 0.36 & 13.35 & 12.50 \\
\hline & & $\mathrm{K}_{3}$ & 2.50 & 2.50 & 0.22 & 0.22 & 0.39 & 0.38 & 13.10 & 13.07 \\
\hline & \multirow{4}{*}{$\mathrm{V}_{2}$} & $\mathrm{~K}_{0}$ & 2.10 & 2.31 & 0.18 & 0.18 & 0.32 & 0.32 & 13.20 & 12.40 \\
\hline & & $\mathrm{K}_{1}$ & 2.60 & 2.50 & 0.20 & 0.20 & 0.38 & 0.36 & 14.40 & 13.30 \\
\hline & & $\mathrm{K}_{2}$ & 2.65 & 2.52 & 0.20 & 0.20 & 0.34 & 0.35 & 14.60 & 13.35 \\
\hline & & $\mathrm{K}_{3}$ & 2.66 & 2.52 & 0.21 & 0.22 & 0.33 & 0.35 & 14.60 & 13.35 \\
\hline \multirow{12}{*}{$\mathrm{I}_{2}$} & \multirow{4}{*}{$\mathrm{V}_{0}$} & $\mathrm{~K}_{0}$ & 2.10 & 2.03 & 0.15 & 0.15 & 0.33 & 0.33 & 11.60 & 11.10 \\
\hline & & $\mathrm{K}_{1}$ & 2.80 & 2.64 & 0.19 & 0.19 & 0.40 & 0.38 & 11.65 & 10.30 \\
\hline & & $\mathrm{K}_{2}$ & 2.87 & 2.15 & 0.20 & 0.20 & 0.42 & 0.40 & 11.90 & 11.20 \\
\hline & & $\mathrm{K}_{3}$ & 2.58 & 2.22 & 0.19 & 0.19 & 0.45 & 0.42 & 11.95 & 11.10 \\
\hline & \multirow{4}{*}{$\mathrm{V}_{1}$} & $\mathrm{~K}_{0}$ & 2.15 & 2.16 & 0.20 & 0.19 & 0.27 & 0.28 & 12.20 & 10.15 \\
\hline & & $\mathrm{K}_{1}$ & 2.90 & 2.60 & 0.21 & 0.22 & 0.32 & 0.30 & 12.53 & 13.20 \\
\hline & & $\mathrm{K}_{2}$ & 2.77 & 2.96 & 0.22 & 0.24 & 0.29 & 0.30 & 14.47 & 13.30 \\
\hline & & $\mathrm{K}_{3}$ & 2.48 & 2.96 & 0.25 & 0.24 & 0.30 & 0.30 & 14.90 & 14.35 \\
\hline & \multirow{4}{*}{$\mathrm{V}_{2}$} & $\mathrm{~K}_{0}$ & 2.10 & 2.10 & 0.18 & 0.18 & 0.27 & 0.26 & 10.35 & 9.40 \\
\hline & & $\mathrm{K}_{1}$ & 2.60 & 2.25 & 0.20 & 0.20 & 0.28 & 0.23 & 10.40 & 10.30 \\
\hline & & $\mathrm{K}_{2}$ & 2.65 & 2.25 & 0.22 & 0.24 & 0.30 & 0.32 & 11.38 & 10.38 \\
\hline & & $\mathrm{K}_{3}$ & 2.60 & 2.30 & 0.25 & 0.24 & 0.35 & 0.35 & 11.43 & 10.45 \\
\hline \multirow{12}{*}{$\mathrm{I}_{3}$} & \multirow{4}{*}{$\mathrm{V}_{0}$} & $\mathrm{~K}_{0}$ & 2.03 & 2.05 & 0.12 & 0.13 & 0.30 & 0.29 & 9.55 & 8.59 \\
\hline & & $\mathrm{K}_{1}$ & 2.30 & 2.31 & 0.21 & 0.20 & 0.41 & 0.40 & 9.60 & 8.66 \\
\hline & & $\mathrm{K}_{2}$ & 2.35 & 2.43 & 0.19 & 0.20 & 0.42 & 0.43 & 10.60 & 8.40 \\
\hline & & $\mathrm{K}_{3}$ & 2.35 & 2.36 & 0.20 & 0.213 & 0.45 & 0.44 & 10.80 & 8.70 \\
\hline & \multirow[t]{4}{*}{ V1 } & $\mathrm{K}_{0}$ & 2.10 & 2.10 & 0.15 & 0.140 & 0.29 & 0.28 & 8.55 & 7.49 \\
\hline & & $\mathrm{K}_{1}$ & 2.31 & 2.31 & 0.16 & 0.157 & 0.31 & 0.30 & 9.40 & 8.40 \\
\hline & & $\mathrm{K}_{2}$ & 2.45 & 2.46 & 0.20 & 0.200 & 0.32 & 0.31 & 9.50 & 8.66 \\
\hline & & $\mathrm{K}_{3}$ & 2.52 & 2.50 & 0.20 & 0.223 & 0.33 & 0.33 & 10.80 & 8.66 \\
\hline & \multirow[t]{4}{*}{$\mathrm{V} 2$} & $\mathrm{~K}_{0}$ & 2.10 & 2.10 & 0.15 & 0.157 & 0.27 & 0.23 & 9.50 & 8.40 \\
\hline & & $\mathrm{K}_{1}$ & 2.38 & 2.35 & 0.17 & 0.177 & 0.34 & 0.34 & 9.60 & 8.40 \\
\hline & & $\mathrm{K}_{2}$ & 2.45 & 2.45 & 0.20 & 0.200 & 0.34 & 0.34 & 10.40 & 8.50 \\
\hline & & $\mathrm{K}_{3}$ & 2.52 & 2.52 & 0.21 & 0.207 & 0.34 & 0.35 & 10.50 & 9.30 \\
\hline \multicolumn{3}{|l|}{ L.S.D at $5 \%$} & 0.020 & 0.020 & 0.01 & 0.01 & 0.020 & 0.020 & 0.11 & 0.14 \\
\hline
\end{tabular}


Table (8): The values of water applied $\left(\mathrm{m}^{3} / \mathrm{Fed}\right)$, wheat grain yield $(\mathrm{Kg} / \mathrm{Fed})$ and field water used water used efficiency $\left(\mathrm{Kg} / \mathrm{m}^{3}\right)$ for different treatments of vitamins soaking during two seasons.

\begin{tabular}{|c|c|c|c|c|c|c|c|}
\hline \multirow{2}{*}{$\begin{array}{l}\text { Irrigation } \\
\text { Frequencies }\end{array}$} & \multirow{2}{*}{$\begin{array}{l}\text { Soaking } \\
\text { in } \\
\text { vitamins }\end{array}$} & \multirow{2}{*}{$\begin{array}{l}\text { Potassium } \\
\text { Application }\end{array}$} & \multirow{2}{*}{$\begin{array}{l}\text { Water } \\
\text { Applied }\end{array}$} & \multicolumn{2}{|c|}{ Grain yield (Kg/Fed.) } & \multicolumn{2}{|c|}{$\begin{array}{c}\text { field water use efficiency } \\
(\mathrm{kg} / \mathrm{m} 3)\end{array}$} \\
\hline & & & & $2005 / 2006$ & $2006 / 2007$ & $2005 / 2006$ & $2006 / 2007$ \\
\hline \multirow{12}{*}{$\mathrm{I}_{1}$} & \multirow{4}{*}{$\mathrm{V}_{0}$} & $\mathrm{~K}_{0}$ & 3000 & 2194.50 & 2161.50 & 0.73 & 0.72 \\
\hline & & $\mathrm{K}_{1}$ & 3000 & 2361.00 & 2200.50 & 0.79 & 0.73 \\
\hline & & $\mathrm{K}_{2}$ & 3000 & 2454.00 & 2281.50 & 0.82 & 0.76 \\
\hline & & $\mathrm{K}_{3}$ & 3000 & 2806.50 & 2604.00 & 0.94 & 0.87 \\
\hline & \multirow{4}{*}{$\mathrm{V}_{1}$} & $\mathrm{~K}_{0}$ & 3000 & 2466.00 & 2437.50 & 0.82 & 0.813 \\
\hline & & $\mathrm{K}_{1}$ & 3000 & 2634.00 & 2491.50 & 0.88 & 0.83 \\
\hline & & $\mathrm{K}_{2}$ & 3000 & 2806.50 & 2632.50 & 0.94 & 0.88 \\
\hline & & $\mathrm{K}_{3}$ & 3000 & 2727.00 & 2761.50 & 0.91 & 0.92 \\
\hline & \multirow{4}{*}{$\mathrm{V}_{2}$} & $\mathrm{~K}_{0}$ & 3000 & 2427.00 & 2481.00 & 0.81 & 0.83 \\
\hline & & $\mathrm{K}_{1}$ & 3000 & 2787.00 & 2602.50 & 0.93 & 0.87 \\
\hline & & $\mathrm{K}_{2}$ & 3000 & 3048.00 & 2920.50 & 1.02 & 0.97 \\
\hline & & $\mathrm{K}_{3}$ & 3000 & 3189.00 & 3168.00 & 1.06 & 1.06 \\
\hline \multirow{12}{*}{$\mathrm{I}_{2}$} & \multirow{4}{*}{$\mathrm{V}_{0}$} & $\mathrm{~K}_{0}$ & 2500 & 1746.00 & 1717.50 & 0.70 & 0.69 \\
\hline & & $\mathrm{K}_{1}$ & 2500 & 1893.00 & 1828.50 & 0.76 & 0.73 \\
\hline & & $\mathrm{K}_{2}$ & 2500 & 2133.00 & 2062.50 & 0.85 & 0.83 \\
\hline & & $\mathrm{K}_{3}$ & 2500 & 2280.00 & 2371.50 & 0.91 & 0.95 \\
\hline & \multirow{4}{*}{$\mathrm{V}_{1}$} & $\mathrm{~K}_{0}$ & 2500 & 2074.50 & 1861.50 & 0.82 & 0.74 \\
\hline & & $\mathrm{K}_{1}$ & 2500 & 2152.50 & 2188.50 & 0.86 & 0.88 \\
\hline & & $\mathrm{K}_{2}$ & 2500 & 2413.50 & 2283.00 & 0.97 & 0.91 \\
\hline & & $\mathrm{K}_{3}$ & 2500 & 2737.50 & 2748.00 & 1.10 & 1.10 \\
\hline & \multirow{4}{*}{$\mathrm{V}_{2}$} & $\mathrm{~K}_{0}$ & 2500 & 2040.00 & 1849.50 & 0.82 & 0.74 \\
\hline & & $\mathrm{K}_{1}$ & 2500 & 2248.50 & 2332.50 & 0.90 & 0.93 \\
\hline & & $\mathrm{K}_{2}$ & 2500 & 2643.00 & 2568.00 & 1.06 & 1.03 \\
\hline & & $\mathrm{K}_{3}$ & 2500 & 2886.00 & 2890.50 & 1.15 & 1.16 \\
\hline \multirow{12}{*}{$\mathrm{I}_{3}$} & \multirow{4}{*}{$\mathrm{V}_{0}$} & $\mathrm{~K}_{0}$ & 2000 & 1792.50 & 1678.50 & 0.90 & 0.88 \\
\hline & & $\mathrm{K}_{1}$ & 2000 & 1801.50 & 1713.00 & 0.90 & 0.86 \\
\hline & & $\mathrm{K}_{2}$ & 2000 & 1848.00 & 1852.50 & 0.92 & 0.93 \\
\hline & & $\mathrm{K}_{3}$ & 2000 & 2130.00 & 2058 & 1.07 & 1.03 \\
\hline & \multirow{4}{*}{$\mathrm{V}_{1}$} & $\mathrm{~K}_{0}$ & 2000 & 1839.00 & 1816.50 & 0.92 & 0.91 \\
\hline & & $\mathrm{K}_{1}$ & 2000 & 1905.00 & 1867.50 & 0.96 & 0.93 \\
\hline & & $\mathrm{K}_{2}$ & 2000 & 2278.50 & 2175.00 & 1.14 & 1.09 \\
\hline & & $\mathrm{K}_{3}$ & 2000 & 2298.00 & 2373.00 & 1.15 & 1.19 \\
\hline & \multirow{4}{*}{$V_{2}$} & $\mathrm{~K}_{0}$ & 2000 & 1867.50 & 1831.50 & 0.93 & 0.92 \\
\hline & & $\mathrm{K}_{1}$ & 2000 & 2172.00 & 2128.50 & 1.09 & 1.06 \\
\hline & & $\mathrm{K}_{2}$ & 2000 & 2388.00 & 2317.50 & 1.19 & 1.16 \\
\hline & & $\mathrm{K}_{3}$ & 2000 & 2511.00 & 2491.50 & 1.26 & 1.25 \\
\hline
\end{tabular}


$I_{1} \times V X K$

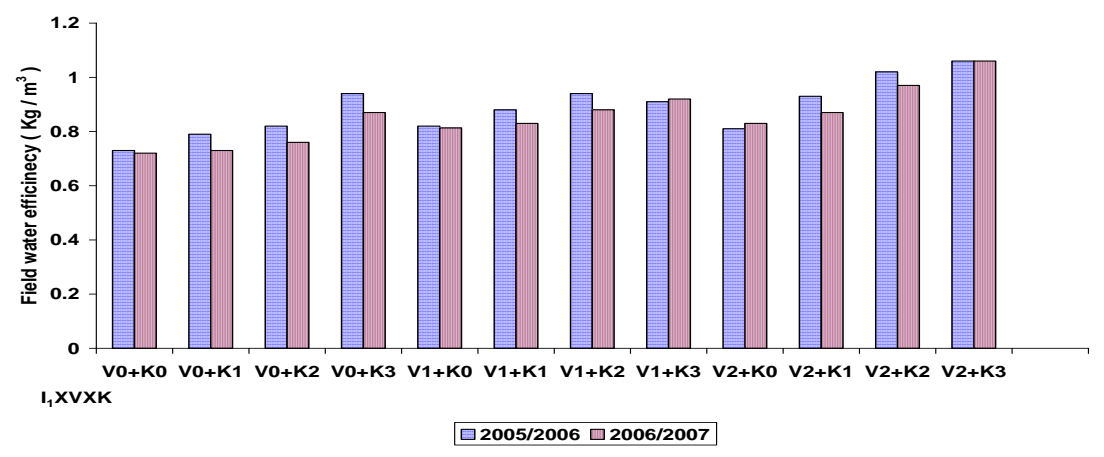

$I_{2} X V X K$

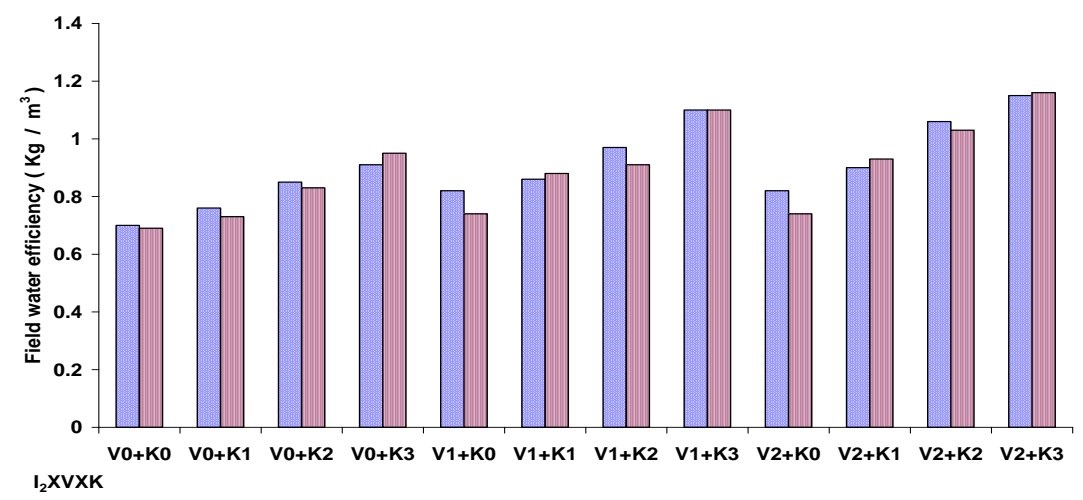

I3XVXK

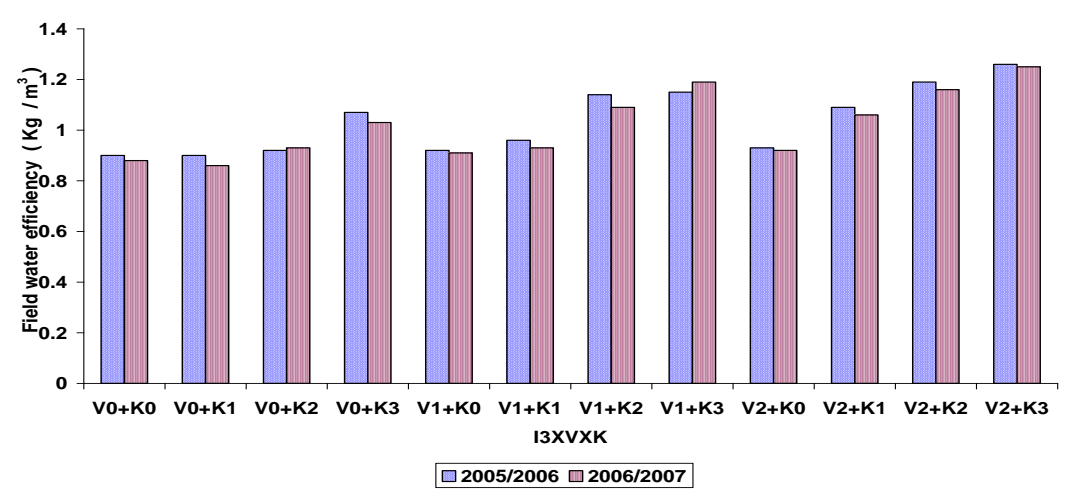

Fig. (1): The values of field water used efficiency $\left(\mathrm{Kg} / \mathrm{m}^{3)}\right.$ for different treatments of vitamins soaking during two seasons. 


\section{Conclusion}

Although the maximum grain yield of wheat was grained from irrigation at 21day interval with soaking in $\mathrm{V}_{2}$ (pyridoxine $\mathrm{B}_{6}$ vitamin) and foliar spray of potassium fertilizer, it can be recommended that irrigation at 28-day interval with soaking in $\mathrm{V}_{1}$ (ascorbic acid $\mathrm{AA}$ ) and foliar spray of potassium fertilizer under Assiut conditions are more suitable according to the following reasons:

1- Spare about $500 \mathrm{~m}^{3}$ from irrigation water per one feddan.

2- The higher contents of macronutrients and total amino acids.

3- The grain yield was only less $2 \mathrm{ard} / \mathrm{Fed}$. than the treatment $\left(\mathrm{I}_{1} \mathrm{~V}_{2} \mathrm{~K}_{3}\right)$ gave the higher grains values.

\section{References:}

1. ABD EL-GAWAD, A.A.; N.A. NOURELDIN, M.A. ASHOUB AND M.S. KASHABA. (1993 ) : Studies on consumptive use and irrigation scheduling in relation to nitrogen fertilization on wheat. II- Response of wheat and its attributes. Annals Agric. Sci., Ain Shams Univ. Cairo, 38 (1): 173-181.

2. ABD-ALLA, A.A. (2002): Effect of seeding rate, phosphorus and potassium fertilization on yield potential of wheat grown under sandy soil conditions. Egypt. J. Apple. Sci., 17 (3): 124-138.

3. ABDEL-AZIZ, EL-SET A. AND U.S. EL-BIALY (2004): Response of maize plant to soil moisture stress and foliar spray with potassium. J. Agric. Mansoura Univ., 29 (6): 3599-3619.

4. ABDEL-HAFEZ, S.A.; S.H. ABOU-KHADRAH; F.A. SUROUR AND A.Z. ELBABLY. (1999): Influence of water stress on some water relations and calculation of potenical evapotranspiration on estimating water requirements. Third Conf. of on farm irrigation and Agroclimatology, 25-27 Jan., Egypt, pp. 477-489.

5. Al-BARRAK,K.M. (2006) : Water use efficiency in wheat grown under drought conditions.J.Biol.Sci.,6(2) :408-411.

6. AZOOZ, M.M. (1990): Physiology studies on the interactive effects of salinity and vitamins on some crop plants. M.Sc. Thesis, Assiut University, Egypt, pp: 1-129

7. BENDER.D.A. ( 1985 ) : Amino acids metabolism. John Wiley Sons.Inc.New York.

8. El-DEFAN, T.A.A.; H.M.A. EL-KHOLI; M.G.M. RIFAAT AND A.E.A. ALLAH. (1999): Effect of soil and foliar application of potassium on yield and mineral content of wheat grains grown in sandy soils. Egypt. J. of Agric. Res., 77 (2): 513-522.

9. El-KHOLY, M.A. (2000): Response of wheat growth and yield to plant density and methods of nitrogen and potassium fertilizer application. Egypt. J. Agric., Vol. 22, pp. 118. 
10. El-SAEI, M.A.; E.M. EL-HADIDI AND A. TALAAT (2006): Water saving by applying potassium fertilization and water stress with wheat crop. J. Agric. Sci. Mansoura Univ., 31 (7): 4825-4835.

11. EL- SAYED M.A A. (2003) : Response of wheat to irrigation in sandy soils. Zagazig.J.Res.,30 (1) : 1-15.

12. El-SAYED, M.M. (2007): Water requirements of wheat and sunflower under different irrigation systems at Assiut. M.Sc. Thesis, Faculty of Agric., Assiut Univ.

13. El-TAYEB, M.A. (1991): Effect of ascorbic acid, pyridoxine or methionine application on some drought stressed crop plants.Ph.D.Thesis, Qena, Assiut Univ., Egypt.1-230.

14. EL-YAMANY, M.S. (1994): Study of the efficiency of some fertilizer treatments on wheat under different irrigation conditions. Ph.D. Thesis, Fac. Agric., Kafer El-Sheikh, Tanta Univ., Egypt.

15. GOMEZ, K.A. AND A.A. GOMEZ. (1984): Statistical Procedures for Agricultural Research. John Wiley and Sons, Inc., New York, U.S.A.

16. HUSSEIN, T.M.; M.E.MELEHA; A.S. ALY AND A.A. NASSER (2000) : The effect of soil moisture levels on irrigation water saving and wheat production.The Eng. Conference of Misr Society of Agri. Eng. Fac. Agric. Minoviya Univ., 25-26 Oct. 2000.

17. HUSSIEN, M.A. (2005): Effect of supplemental irrigations, seeding rates and foliar application of potassium and macro-micro elements on wheat productivity under rain fed conditions. Bull. Fac. Agric., Cairo Univ., 56: 431-454.

18. IBRAHIM, M.E.; H.M.M. EL-NAGGAR AND A.A. EL-HOSARY (1992): Effect of irrigation intervals and plant densities on some varieties of corn. Menofiya J. Agric. Res., 17: 1083-1098.

19. JACKSON , M.L. (1970) : Soil chemical analysis . Prentice-Hall Inc., Englewood cliffs, N.J., U.S.A.

20. KANSAL, B.D. AND G.S. SOKHON (1973): Influence of potassium application on dry matter yield and cationic balance in wheat. Agro., 18: 94-101.

21. KLUTE, A. (1986): Methods of Soil Analysis. Part 1. Physical and Mineralogical methods. 2nd ed. Agron. Monogr. No. 9. ASA and SSA, Madison, WI.

22. MAHDY, E.E AND F.A.TEAMA (2000): Response of some wheat cultivars to fertilization and seeding rate in new reclaimed and clay soil in Upper Egypt. BMorphological trails and grain protein percentage .Assiut J.Agric.Sci:135 -148.

23. MARSCHNER, H. (1995): Mineral Nutrition of Higher Plants. 2nd ed. Academic Press, London, San Diego, New York, Boston, Sydney, Tokyo, Toronto.

24. MOHAMED, S.A. AND A.M.M. ALI (1982): Influence of potassium dose on water economy for winter wheat in Fayoum Governorate Research Bulletin, Fac. Agric., AinShams Univ.: 22-30. 
25. MOORE,S AND W. STEIN (1948): Photometric of ninhydrine method for used in the chromatography of amino acid .From the laboratories of Rockefeller Institute For Medical Research, New York.

26. PAGE, A.L. (1982): Methods of Soil Analysis. Part 1, Chemical and Microbiological properties. 2nd ed. Soil Sci. Soc. Am. Inc., Madison, USA.

27. RAGHEB, H.M.A. AND G. EL-NAGAR. (1997): Response of grain sorghum to nitrogen fertilization and irrigation intervals. Assiut J. Agric. Sci. 28 (2): 145-157.

28. SAAD, A.M.; A.T. THALOOTH AND H.A. EL-ZEINY. (1990): Late foliar fertilization with $\mathrm{N}, \mathrm{P}$ and $\mathrm{K}$ for increasing yield and protein content of wheat grains. Egypt. J. Agron., 15 (1-2): 217-228.

29. SHADDAD, M.A., A.F. RADI , A.M. ABDEL-RAHAMAN AND A.A. AZOOZ. (1990): Response of seeds of Lupinus termis and Vicia faba to the interactive effect of salinity and ascorbic acid or pyridoxine (B6). Plant and Soil, 122: 177-186.

30. TALHA, M.; M. EL-TONY AND A. ABO GABAL. (1987 ): The effect of soil water deficit on yield of wheat and evapotranspiration. Egypt. J. Soil Sci. 27 (1): 63-73.

31. ZIDAN,M.A.(1990) : Alleviation of salinity stress on growth and related parameters in wheat sprayed with thiamine, nicotinic acid and pyridoxine.Arab.Gulf.J.Scient.Res.9 : 103-117. 